\title{
STABILISATION DES RÉSIDUS SOLIDES DE L'INCINÉRATION PAR LE PROCÉDÉ TREDI-ASH ÉTAPE 1 : TRAITEMENT DES MÂCHEFERS
}

\author{
Danielle Venditti*, Sylvain Durécu*, Jacques Thauront**, Guy Fraysse***, Jacques Berthelin**** \\ * Laboratoire de recherche Tredi, ** Direction générale de Tredi, *** Centre Tredi de Saint-Vulbas, **** Centre de pédologie biologique, UPR 683I du CNRS
}

\begin{abstract}
La législation relative à la mise en décharge des mâchefers d'incinération de déchets industriels imposera dans un avenir proche la stabilisation de ces résidus comme préalable au stockage. Le procédé Tredi-ASH transforme par lixiviation chimique une fraction pondérale importante des scories traitées en résidus stables (70 à $95 \%$ du poids $\mathrm{sec})$. Les matières en suspension générées par le brassage mécanique ( 5 à $30 \%$ du poids sec initial) sont séparées par filtration et peuvent être recyclées sur les unités d'incinération, en pré-neutralisation des eaux acides issues du lavage des fumées. Les métaux toxiques extraits dans les lixiviats sont traités par biosorption microbienne. Cette seconde partie du procédé fera l'objet d'un prochain article.
\end{abstract}

Incineration of industrial wastes produces slags which, according to the future french regulations, will require to be stabilized prior to disposal. Portions of heavy metals contained in slags could indeed be solubilized and therefore constitute a toxic mobile fraction which has to be eliminated. Tredi-ASH is a chemical leaching process (exchange reaction with $\mathrm{CaCl}_{2}$, obtained from wet gas cleaning neutralization) used to extract mobile metal pollutants from slags. This process allows to detoxify and to stabilize an important fraction of slag's original weight (70 to $95 \%$ dry weight), reducing ultimate waste volume without transfer of pollution. Sludges generated by stirring and leaching (5 to $30 \%$ dry weight) can be recycled on waste incineration plants, to neutralize acid waters originating from wet gas cleaning.

Au ler Juillet 2002, seuls les déchets ultimes pourront être stockés, la législation imposant dés 1998 la stabilisation des mâchefers d'incinération de déchets industriels avant leur admission en CET. En effet, ces scories, comme les MIOM (mâchefers d'incinération d'ordures ménagères), contiennent certains éléments potentiellement polluants (métaux lourds, sulfates...) dont la mobilité doit être évaluée, et si nécessaire réduite (Wicker et al., 1995 ; Jozon \& Troesch, 1995).

Le procédé Tredi-ASH, permettant de transformer une fraction pondérale importante des résidus solides de l'incinération en déchets stables, a été développé pour satisfai- re à cette échéance.

La réaction chimique permettant de solubiliser les métaux mobilisables des mâchefers est une réaction d'échange cationique, qui met en œuvre le chlorure de calcium produit au cours de l'épuration des fumées par voie humide dans les unités d'incinération. Le calcium se fixe sur les scories, en libérant les métaux labiles dans la solution, selon le schéma simplifié de la figure I. Cette lixiviation chimique est couplée à un brassage mécanique, qui en accentue l'efficacité. Le calcium fixé permet, dans un second temps, d'accentuer la carbonatation naturelle des résidus traités (maturation par stockage à l'air).

Les matières en suspension résiduelles générées par la lixiviation ( 5 à $30 \%$ de la masse sèche initiale) peuvent être recyclées en pré-neutralisation des eaux acides issues du lavage des gaz, lorsque leur qualité et leur teneur en carbonates (après maturation, si nécessaire) le permettent.

Les solutions de chlorure de calcium enrichies en métaux sont traitées par biosorption microbienne (Étape 2 : Traitement des lixiviats, dans un prochain numéro). Les biomasses chargées en polluants peuvent être régénérées ou réduites en cendres.

Les essais à l'échelle semi industrielle ont porté sur plus de 12 tonnes de scories, échantillonnées ponctuellement, différentes en qualité et issues des différents centres d'incinération Tredi (Saint-Vulbas, Salaise \& Strasbourg).

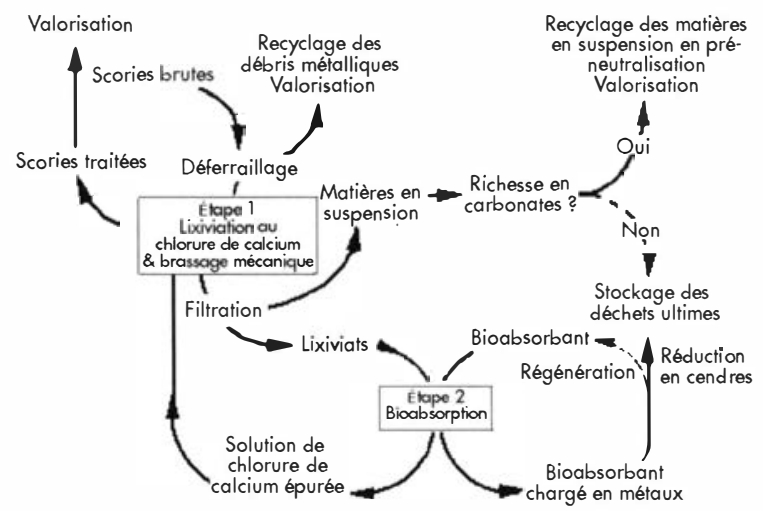

Figure I : Schéma simplifié des deux étapes du traitement par le procédé Tredi-ASH 


\section{RÉSULTATS OBTENUS}

\section{Traitement des scories}

\section{Les métaux lourds labiles échangés}

Le tableau I présente les bilans massiques des métaux suivis, rapportés pour le traitement de I kg de mâchefers (lot issu du centre de Saint-Vulbas). Le suivi analytique a porté sur les 3 métaux lourds présents en quantités prépondérantes dans la scorie brute : le zinc, le plomb et le cuivre. Ces métaux lourds représentent initialement $0,4 \%$ de la masse sèche des mâchefers.

La remontée du pH de l'effluent observée au cours du traitement est imputable à la nature même des mâchefers d'incinération de déchets industriels, souvent basiques comme les MIOM (Wicker et al., 1995).

Lors de la réaction d'échange, les métaux sont extraits des scories et sont transférés dans l'effluent industriel. Toutefois, le $\mathrm{pH}$ alcalin de l'effluent favorise la formation d'hydroxydes métalliques peu solubles (zincate, plombite...) qui sont, en partie, retenus dans l'étape de séparation des fines par filtration à $3 \mu \mathrm{m}$. Ces métaux sous forme d'hydroxydes sont mis en solution par $\mathrm{LiBO}_{2}-\mathrm{HNO}_{3}$ avant le dosage en ICP-MS, de la même façon que les fines, même s'ils n'en font pas partie intégrante.

Le traitement permet l'extraction dans les matières en suspension ( $5 \%$ du poids sec initial) et dans les hydroxydes métalliques retenus avec elles, de $11,7 \%$ des métaux suivis (plomb, zinc et cuivre). Ce pourcentage est représentatif de la fraction de ces métaux labiles dans les mâchefers, les 86,3 \% restants correspondant aux métaux peu mobiles, piègés dans la matrice des scories. Le traitement aboutit à l'obtention de 91,5\% de scories traitées (en poids sec), les résultats tenant compte de la perte de masse relative à la perte de fraction soluble des résidus ( $1,75 \%)$.

Les fines associées aux hydroxydes métalliques renferment $15,2 \%$ du plomb total initial, $12,7 \%$ du cuivre total initial, et

Tableau I : Bilan massique rapporté à I kg de mâchefers (lot de Saint-Vulbas) traité par le procédé Tredi-ASH. Lixiviation de $2 \mathrm{H}$, ratio Solide/Liquide : I/2, pH final de l'efiluent industriel : I I,2.

\begin{tabular}{|c|c|c|c|c|}
\hline & $\begin{array}{l}\text { Poids } \\
\text { sec (g) }\end{array}$ & $\begin{array}{c}\text { Plomb } \\
\text { (mg) }\end{array}$ & $\begin{array}{c}\text { Cuivre } \\
\text { (mg) }\end{array}$ & $\begin{array}{l}\text { Zine } \\
(\mathbf{m g})\end{array}$ \\
\hline $\begin{array}{l}\text { Scories avant traitement } \\
\text { Quantité totale de métal' } \\
\text { Quantité mobilisable par lixiviation norma- } \\
\text { lisée, pH moyen des } 3 \text { lixiviats : } 10,75^{2}\end{array}$ & 1000 & $\frac{1150}{42}$ & $\begin{array}{l}1005 \\
8,20\end{array}$ & 2350 \\
\hline $\begin{array}{l}\text { Scories après traitement } \\
\text { Quantité totale de métal' } \\
\text { Quantité mobilisable par lixiviation norma- } \\
\text { lisée, pH moyen des } 3 \text { lixiviats : } 10,4^{2}\end{array}$ & 915 & 949 & 844 & 2095 \\
\hline $\begin{array}{l}\text { Matières en suspension } \\
\text { (filtration à } 3 \mu \mathrm{m} \text { ) } \\
\text { Quantité totale de métal' } \\
\text { Quantité mobilisable par lixiviation norma- } \\
\text { lisée, pH moyen des } 3 \text { lixiviats : } 9,8^{2} \\
\text { Effluent filtré, } 2000 \mathrm{ml} \text {, pH } 1 \mathrm{I}, 2^{2}\end{array}$ & 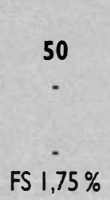 & $\begin{array}{l}174,90 \\
1,50 \\
0,70\end{array}$ & $\begin{array}{l}127,90 \\
0,76 \\
0,44\end{array}$ & 222,4 \\
\hline
\end{tabular}

$10,6 \%$ du zinc total initial. Ce dernier est vraisemblablement piégé dans des complexes plus stables au sein de la matrice de la scorie, comme l'indiquent aussi les résultats de la lixiviation normalisée (tableau I).

Les faibles valeurs obtenues pour les dosages de l'effluent industriel et des eaux de lixiviation normalisée sont à interpréter en relation avec la basicité de leurs $\mathrm{pH}$, et avec la procédure analytique utilisée pour le dosage des métaux en solution. En effet, avant d'être dosés en ICP-AES, les échantillons sont filtrés à $0,45 \mu \mathrm{m}$, par référence à la norme $X 31$ 210 : les hydroxydes métalliques piégés sur le filtre ne peuvent être dosés.

Cette sous estimation à l'analyse des teneurs en métaux des liquides n'est pas négligeable, si l'on se réfère à la congruence des bilans massiques (l'effluent filtré devrait contenir quelques $12 \mathrm{mg} / \mathrm{l}$ de plomb, $16 \mathrm{mg} / \mathrm{l}$ de cuivre et 15 $\mathrm{mg} / \mathrm{l}$ de zinc), et est certainement accentuée dans l'effluent par rapport aux eaux météoriques, en raison de la présence d'autres espèces chimiques en concentrations importantes.

Nonobstant cette évidente inadéquation entre les dosages en solution et le bilan des analyses sur déchet sec, il apparaît clairement que la fraction métallique mobilisable à l'eau (estimée) est réduite au terme de la réaction d'échange, d'un facteur 5 pour le plomb, et 3 pour le cuivre.

Pour démontrer l'efficacité de la réaction d'échange par rapport à un simple brassage à l'eau, d'autres essais ont été réalisés sur un lot de mâchefers de faible basicité issus du centre de Salaise. Trois essais ont été réalisés sur ce même lot de scories (tableaux $2 \mathrm{a}$ et $2 \mathrm{~b}$ ) :

- $200 \mathrm{~kg}$ ont été traités par un effluent salin industriel prélevé en sortie du clarificateur du centre Tredi Saint-Vulbas (Essai A),

- $200 \mathrm{~kg}$ ont été traités par un effluent salin industriel prélevé en sortie du clarificateur du centre Tredi Salaise (Essai B),

- $200 \mathrm{~kg}$ ont été traités par de l'eau industrielle (Essai C). Pour les 3 essais, le pH des liquides reste voisin de 8 au terme de la lixiviation, c'est à dire que le phénomène de perte d'hydroxydes métalliques à l'analyse est réduit.

La réaction d'échange est facilitée lorsque la concentration en calcium augmente (tableaux $2 \mathrm{a} \& 2 \mathrm{~b}$ ) : l'enrichissement de l'effluent usé en métaux totaux est plus important pour l'essai $B$ qu'il ne l'est pour les essais $A$ et $C$. De la même façon, l'effluent usé $B$ est appauvri de façon plus conséquente en calcium que ne le sont l'effluent $A$ et l'eau. Par ailleurs, les résidus issus du traitement par l'effluent le plus chargé en calcium (essai B) sont plus stables à la lixiviation normalisée, puisque ayant subit une réaction d'échange efficace.

La réaction d'échange, combinée au brassage mécanique, est donc plus efficace pour l'extraction des métaux labiles et la stabilisation des résidus qu'un simple brassage à l'eau.

\section{Cinétique de la réaction de stabilisation}

Cette cinétique est rapide. Sur la base de I,5 tonne de scories issues du centre de Saint-Vulbas traitées lors de 6 cycles 
Tableau 2a: Caractérisation des efiluents utilisés pour le traitement d'un lot de mâchefers issu du centre de Salaise par le procédé Tredi ASH (essais A, B, C).

\begin{tabular}{|c|c|c|c|c|}
\hline & $\mathrm{pH}$ & $\begin{array}{c}\text { Teneur en } \\
\text { MeS (g de } \\
\text { matière } \\
\text { sèchell) }\end{array}$ & $\begin{array}{l}\text { Teneur en } \\
\text { calcium } \\
(\mathrm{mg} / \mathrm{l})\end{array}$ & $\begin{array}{c}\text { Teneur en } \\
\text { métaux totaux } \\
(\mathrm{Pb}, \mathrm{Zn}, \mathrm{Cu}, \mathrm{Cd} \\
\mathrm{Cr}, \mathrm{Ni})(\mathrm{mg} / \mathrm{l})\end{array}$ \\
\hline $\begin{array}{l}\text { Effluent } A \\
\text { Effluent } A \text { après } 2 \\
\text { heures de lixiviation }\end{array}$ & 7,8 & 51,30 & 765 & 0,11 \\
\hline $\begin{array}{l}\text { Effluent } B \\
\text { Effluent } B \text { après } 2 \\
\text { heures de lixiviation }\end{array}$ & 7,6 & 45,40 & $\begin{array}{l}1009 \\
906\end{array}$ & $\begin{array}{l}0,10 \\
5,99\end{array}$ \\
\hline $\begin{array}{l}\text { Effluent } C \\
\text { Effluent } C \text { après } 2 \\
\text { heures de lixiviation }\end{array}$ & $\begin{array}{l}7,1 \\
7,9\end{array}$ & 50,50 & $\begin{array}{l}271 \\
229\end{array}$ & $\begin{array}{l}0,04 \\
0,91\end{array}$ \\
\hline
\end{tabular}

Tableau $2 b$ : Extraction de la fraction métallique par lixiviation normalisée $X 31210$ à l'eau météorique d'un lot de mâchefers issu du centre Tredi de Salaise, avant et après traitement par le procédé Tredi ASH (essais A, B, C) (mglkg sec)

Plomb Cadmium Zinc Cuivre Nickel Chrome Métaux

\begin{tabular}{|c|c|c|c|c|c|c|c|}
\hline & Plom & admiu & Zinc & Cuivr & Nicke & Chrom & $\begin{array}{l}\text { Métaux } \\
\text { totaux }\end{array}$ \\
\hline Scories brutes & 0,99 & 0,29 & 21,50 & 15,90 & 14,30 & 0,09 & 53,1 \\
\hline $\begin{array}{l}\text { Scories traitées } \\
2 \mathrm{H} \text {, Essai A }\end{array}$ & 1,07 & 0,10 & 3,11 & 1,78 & 3,37 & 0,10 & 9,53 \\
\hline $\begin{array}{l}\text { Scories traitées } \\
2 \mathrm{H} \text {, Essai } B\end{array}$ & 0,70 & 0,04 & 2,83 & 0,98 & 2,96 & 0,14 & 7,65 \\
\hline $\begin{array}{l}\text { Scories traitées } \\
2 \mathrm{H} \text {. Essai C }\end{array}$ & 1,42 & 0,14 & 3,88 & 1,82 & 6,18 & 0,19 & 13,63 \\
\hline
\end{tabular}

de lixiviation (figure 2), le seuil d'élution en métaux totaux $(\mathrm{Pb}, \mathrm{Cd}, \mathrm{Zn}, \mathrm{Ni}, \mathrm{Cu}$ et $\mathrm{Cr}$ ) mesuré lors du test normalisé $\times 31210$ est considérablement diminué dés les 30 premières minutes du traitement. Ainsi, $100 \%$ des scories brutes présentent initialement un seuil d'élution en métaux totaux compris entre 5 et $15 \mathrm{mg} / \mathrm{kg}$ de résidu sec. Cette proportion est réduite à $26 \%$ après 30 min de traitement, et atteint un taux inférieur à $20 \%$ après 2 heures. Plus de $80 \%$ des scories présentent alors un seuil inférieur à $5 \mathrm{mg} / \mathrm{kg}$ de résidu sec, répartis majoritairement dans la tranche 2 à 5 $\mathrm{mg} / \mathrm{kg} \mathrm{sec}(67 \%)$, $14 \%$ des scories traitées ayant un seuil

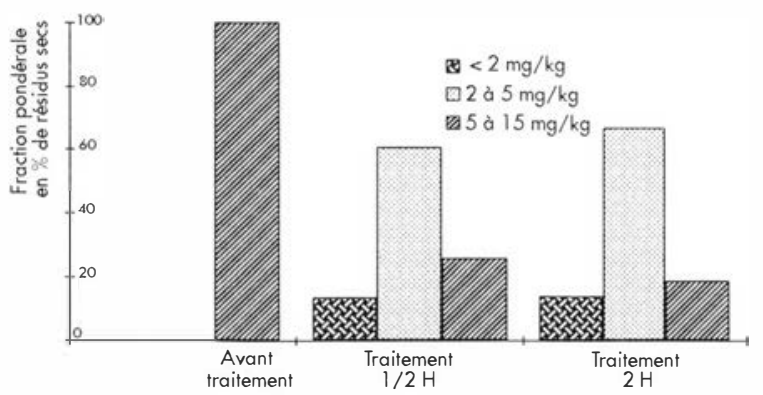

Figure 2 : Résultats obtenus sur 6 cycles de traitement, portant sur un tonnage global de I,5 tonne de scories issues du centre de Saint-Vulbas. Étude de la répartition globale des résidus secs obtenus après traitement. Évolution du seuil de lixiviation pour les métaux lourds $\mathrm{Pb}+\mathrm{Zn}+\mathrm{Cd}+\mathrm{Cu}+\mathrm{Ni}+\mathrm{Cr}$ (exprimés en $\mathrm{mg} / \mathrm{kg}$ de déchets secs) en fonction de la durée du traitement de stabilisation d'élution en métaux totaux inférieur à $2 \mathrm{mg} / \mathrm{kg}$ de matière sèche.

\section{Stabilisation de la matrice des résidus}

Le calcium et le magnésium échangés fixés aux scories après le traitement, ainsi que le fractionnement des résidus par le brassage mécanique, contribuent à en accentuer la carbonatation naturelle; la teneur en carbonates des scories brutes augmente de $5 \%$ après trois mois de maturation naturelle à l'air libre, celle des scories traitées étant incrémentée de $10 \%$ au cours de la même période.

\section{Qualité et devenir des fines générées par brassage mécanique}

Le brassage mécanique exercé par le tambour rotatif, induit la formation de fines particules qui sont sous-tirées par filtration lors du traitement. Les mesures de perte au feu à $550^{\circ} \mathrm{C}(\mathrm{PF})$ sur les scories traitées (lot Saint-Vulbas) montrent une diminution de la teneur en imbrûlés totaux (de nature organique et inorganique) de ces dernières, cette diminution étant particulièrement sensible pendant la première heure de traitement (figure 3 ).

Cette diminution de la teneur en imbrûlés totaux est consé-

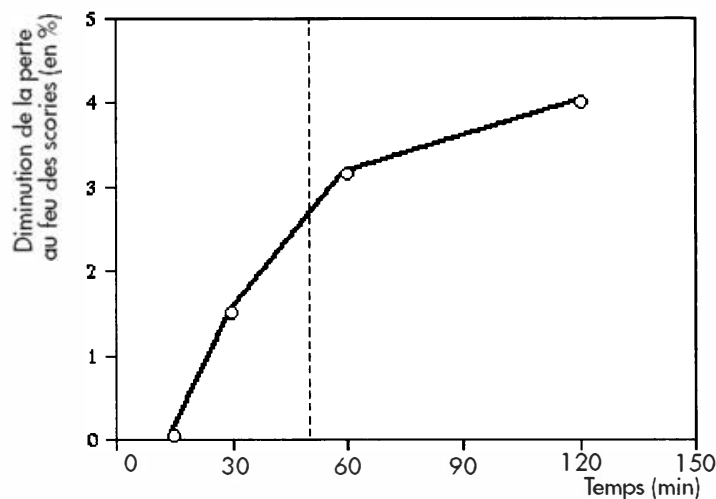

Figure 3 : Étude de la corrélation entre la diminution de perte au feu des scories et le temps de lixiviation, au cours du traitement d'un lot de mâchefers issus du centre de Saint-Vulbas

cutive à la formation des matières en suspension lors du brassage. Le brassage progressif des scories a en effet pour conséquence l'augmentation du ratio PF des fines/PF des scories traitées, en fonction du temps (figure 4).

Les PF des fines générées lors du traitement de scories issues du centre de Strasbourg sont de l'ordre de $5 \%$ (figure 5). La pertinence de ce paramètre $\mathrm{PF}$ à $550^{\circ} \mathrm{C}$ comme témoin de la rémanence d'imbrûlés de nature organique est à discuter : comme le montre la Figure 5 , la mesure de la PF à $550^{\circ} \mathrm{C}$ prend en compte la fraction d'imbrûlés de nature organique $(2,2 \%)$, mais aussi une fraction non organique $(2,4 \%)$ constituée probablement en partie par des carbonates, ceux-ci pouvant être partiellement altérés à $550^{\circ} \mathrm{C}$, en fonction des conditions expérimentales (teneur en oxygène du four réfractaire, proportion de carbonates dans la composition globale...). 


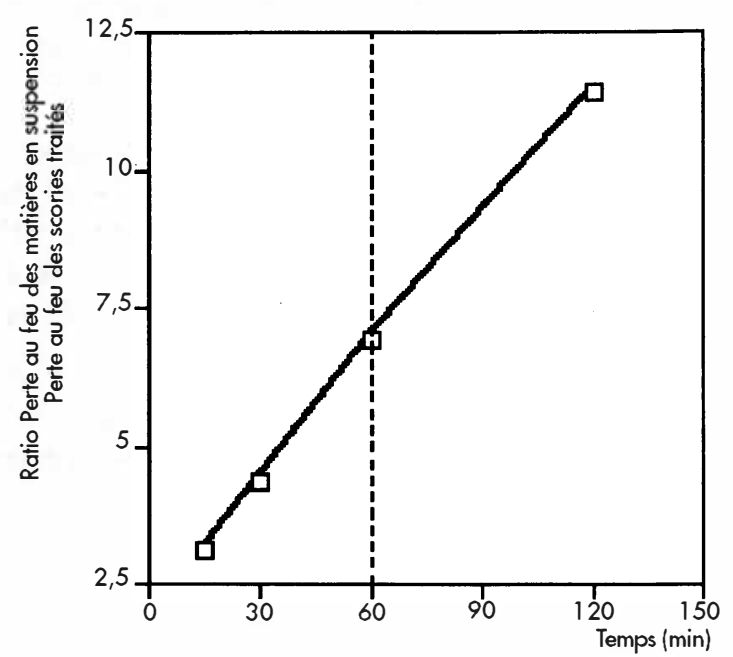

Figure 4 : Étude de la correlation entre le ratio PF fines/PF scories traitées et le temps de lixiviation au cours du traitement d'un lot de scories issues du centre de Saint-Vulbas

Selon la qualité initiale des scories, la teneur en carbonates (déterminée sur la base de l'hypothèse selon laquelle tout le carbone inorganique dosé est présent sous forme de carbonates) peut atteindre 5 à $45 \%$ de la masse sèche des matières en suspension $(42,5 \%$ dans la seule fraction hors PF en figure 5), ce qui permet d'envisager la réutilisation de ces dernières dans le procédé de pré-neutralisation $(\mathrm{pH} 2$ à 5) des eaux acides issues du lavage des fumées. Par ailleurs, le comportement de ces matières en suspension à la lixiviation normalisée est souvent satisfaisant (tableau I).

Ce type d'approche est déjà utilisé sur les centres Tredi : le procédé High Density Sludge (HDS), permet un recyclage

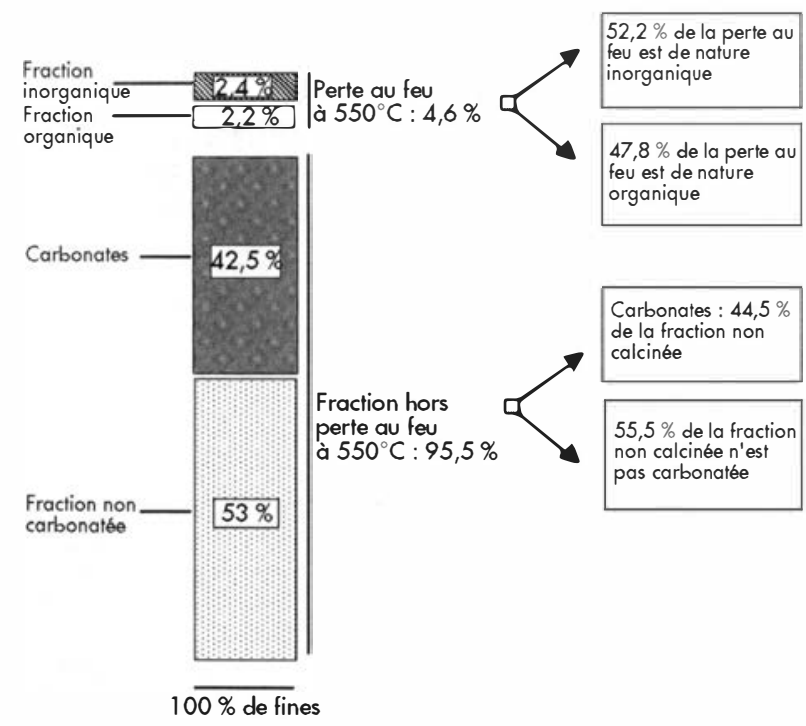

Figure 5 : Histogramme illustrant la composition globale des matières en suspension (fines) générées lors de la stabilisation de mâchefers issus du centre de Strasbourg (valeurs moyennes obtenues sur les fines issues de 4 essais de traitement, portant chacun sur 200 kg de mâchefers) partiel de boues en pré-neutralisation.

La fraction pondérale de fines générées au cours du traitement est corrélée à l'hétérogénéité des scories brutes soumises au traitement de stabilisation : lorsque les mâchefers bruts sont de qualité inférieure, les fines sont plus importantes en quantité (jusqu'à $30 \%$ de la masse sèche initiale), peu riches en carbonates ( 5 à $10 \%$ seulement de la masse sèche), et parfois peu satisfaisantes à la lixiviation normalisée. Dans ce cas de figure, ces matières en suspension sont considérées comme des déchets ultimes et sont destinées au stockage. Inversement, les résultats obtenus dans le cadre de nos essais suggèrent que lorsque la quantité de fines générées est réduite ( $5 \%$ de la masse sèche initiale) car les scories brutes sont de meilleure qualité, la charge en carbonates est importante ( $45 \%$ sur $\mathrm{sec}$ ), les matières en suspension peuvent alors être utilisées en pré-neutralisation.

\section{Les test de lixiviation et l'aspect normatif}

Le lot de scories brutes (Salaise et Saint-Vulbas) ayant fait l'objet de cette étude est conforme à la future législation sur les déchets ultimes (figure 6a) mais n'est pas admissible dans les classes « Maturation 》 ou « Valorisation » définies par la législation sur les MIOM (figure 6b), en raison de dépassements de normes pour les paramètres Fraction Soluble, Sulfates et Plomb.

Au terme du traitement de stabilisation, ces scories sont valorisables en travaux publics (figure 7), puisque les seuils d'élution à la lixiviation normalisée pour les différents critères testés sont inférieurs aux normes imposées pour la classe Valorisation.

Sur la base des essais semi-industriels menés sur plus de 12 tonnes de scories, le procédé Tredi-ASH permet la valorisation (hypothétique) de 70 à $95 \%$ (en masse sèche) des mâchefers, en fonction de la qualité initiale du matériau traité, en agissant sur les fractions métalliques, anioniques et organiques solubilisables grâce à l'effet combiné de la réaction chimique d'échange cationique et du brassage mécanique.

Cette valorisation est hypothétique en ce sens qu'elle ne pourra être rendue effective qu'au terme d'une évolution de la législation. Dans l'état actuel de la réglementation en effet, la valorisation des scories d'incinération de déchets industriels n'est pas autorisée, en dépit de l'innocuité et de la qualité de certains de ces résidus.

\section{CONCLUSIONS}

Le procédé Tredi-ASH met en œuvre un technique physico-chimique de détoxication des résidus de l'incinération dont l'objectif est de stabiliser une fraction importante de mâchefers (70 à $95 \%$ de la matière sèche, selon la qualité initiale des scories traitées) de façon à les rendre idoines à la valorisation comme matériaux de génie civil, et à réduire le volume du déchet ultime à mettre en décharge (dans le cas de figure le moins optimiste, seuls $30 \%$ de la masse sèche initiale - fines + biosorbant chargé réduit en cendres - sont destinés au stockage) et ce sans transfert de pollution. 


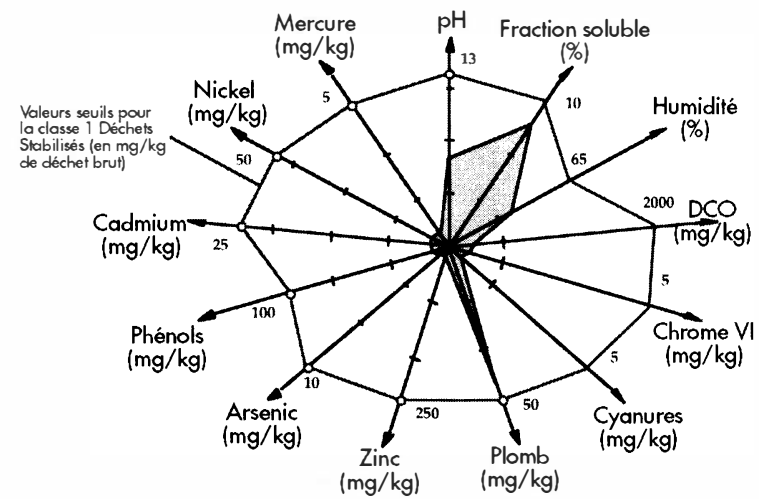

Figure 6a: Analyse de scories brutes produites par le centre de Salaise. Le profil obtenu lors du test de lixiviation normalisé $X 31210$ permet de classer ces mâchefers dans la catégorie « Déchets Stabilisés ", par référence aux valeurs seuils imposées par la réglementation relative aux déchets industriels (arrêté du 18 février 1994)

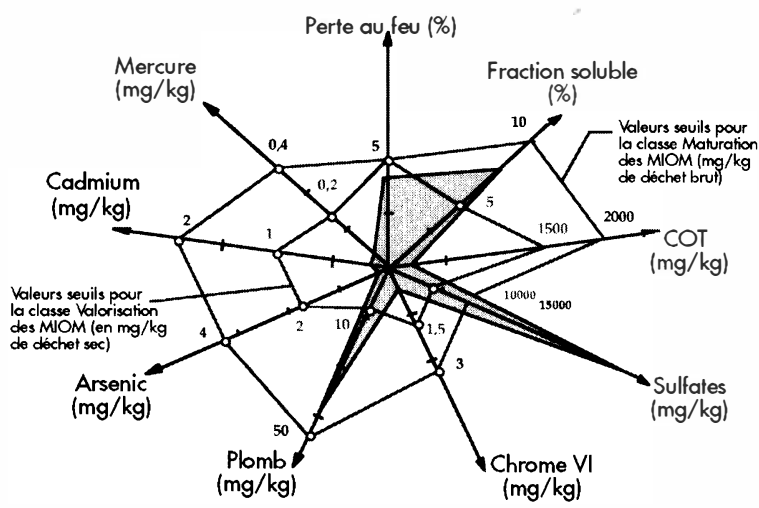

Figure 6b : Par contre, les scories brutes ne sont pas admissibles dans les classes « Maturation » et "Valorisation » définies par la législation relative aux mâchefers d'incinération d'ordures ménagères (MIOM), les paramètres Fraction Soluble et Sulfates étant supérieurs aux valeurs seuils imposées par la réglementation

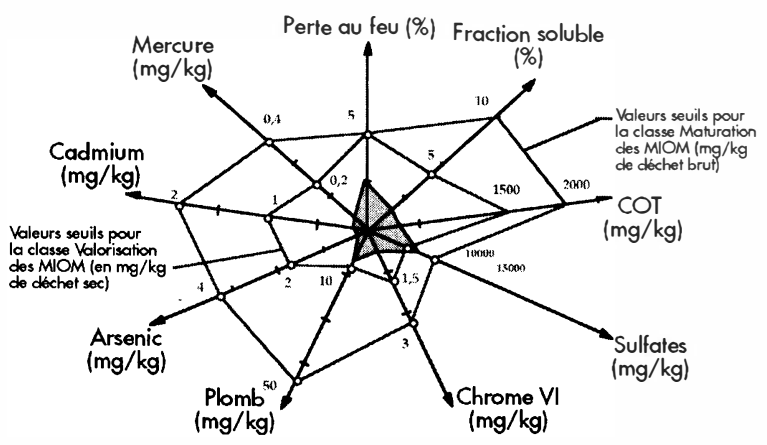

Figure 7 : Scories traitées selon le procédé Tredi ASH. Les seuils d'élution obtenus sont inférieurs aux valeurs réglementaires imposées par la classe «Valorisation » relative aux scories d'incinération d'ordures ménagères (MIOM) ; ces mâchefers sont donc valorisables en travaux publics. Ce profil est à comparer à celui décrit en figure 6 b.
Les fines générées au cours du traitement ( 5 à $30 \%$ de la masse sèche initiale selon la qualité initiale des scories traitées) peuvent être utilisées en pré-neutralisation des eaux acides issues du lavage des fumées, lorsqu'une charge suffisante en carbonates (après maturation si nécessaire) le permet. Dans le cas des fines, le paramètre $\mathrm{PF}$ à $550^{\circ} \mathrm{C}$ prend en compte une fraction inorganique non négligeable, probablement composée en partie de carbonates. II n'est donc pas pertinent à lui seul pour témoigner de la rémanence d'imbrûlés de nature organique dans ces résidus. Seule la détermination des teneurs en carbone organique est probante.

Sur la base des essais semi-industriels menés sur plus de 12 tonnes de scories, 70 à $95 \%$ (en masse sèche) des mâchefers deviennent admissibles en classe Valorisation (législation relative aux déchets urbains) au terme du traitement par le procédé Tredi-ASH.

Les lixiviats chargés en métaux issus de la première étape du traitement peuvent être épurés par biosorption microbienne et sont dès lors réutilisables pour une nouvelle lixiviation de scories car toujours suffisamment chargés en calcium (Étape 2 : Traitement des lixiviats dans un prochain numéro). La biomasse chargée en métaux issue de la seconde étape du traitement peut être régénérée ou réduite en cendres. Le résidu ultime issu de cette incinération est alors stocké.

Le procédé Tredi-ASH aboutissant à une détoxification des scories traitées contribue ainsi à une meilleure protection de notre environnement en limitant les risques induits par la présence de métaux labiles dans ces résidus.

* Danielle Venditti, Sylvain Durécu

Tredi, Laboratoire de recherche, BP 184, 5450I, Vandœurre-lès-Nancy

** Jacques Thauront

Tredi, Direction générale, 62, rue Jeanne d'Arc, 7564I Paris Cedex 13

*** Guy Fraysse

Centre Tredi de Saint-Vulbas, BP 55, 0 I I 50 Lagnieu

**** Jacques Berthelin

Centre de pédologie biologique, UPR 6831 du CNRS, BP 5, 5450 I, Vandœuvre-lès-Nancy.

\section{Bibliographie}

I. Jozon, C. \& O. Troesch (1995). Des exemples de traitement et de valorisation des MIOM face à la nouvelle réglementation. TSM, 5, 40I-403.

2. Wicker R., Blanchard J.-M., \& J. Veron (1995). Caractérisation physicochimique des mâchefers d'incinération de déchets par percolixiviation. TSM, 4, 369374.

3. Thauront J., Durécu S., Fraysse G., Deneux-Mustin S. \& J. Berthelin (1994). Process converts incineration slag into stabilized residues. Environmental Solutions, 12, 42-43. 